\title{
Quel usage les adultes font-ils de la répétition ? Comparaison entre un corpus d'interactions verbales adultes/adolescents polyhandicapés et adultes/jeunes enfants
}

\author{
Christine Bocéréan \\ Université Nancy 2, Laboratoire de Psychologie \\ Christine.Bocerean@univ-nancy2.fr \\ Emmanuelle Canut \\ Université Nancy 2, ATILF \\ Emmanuelle.Canut@univ-nancy2.fr \\ Michel Musiol \\ Université Nancy 2, Laboratoire de Psychologie \\ Michel.Musiol@univ-nancy2.fr \\ Virginie André \\ Université Nancy 2, ATILF \\ Virginie.André@univ-nancy2.fr
}

\section{Introduction}

La recherche présente non seulement l'originalité de s'intéresser aux personnes polyhandicapées francophones mais également de comparer des interactions verbales entre d'une part des adultes et des adolescents polyhandicapés et d'autre part des adultes et des jeunes enfants de même âge mental que les adolescents. Dans les deux corpus, nous avons remarqué et analysé une stratégie communicationnelle particulière de la part de l'adulte, basée sur le phénomène de répétition. Nous avons déjà montré en quoi elle contribue au processus général d'intercompréhension (Musiol, Trognon, Coulon \& Bocéréan, 2006; Verhaegen, Bocéréan \& Musiol, 2008). Dans le cas des interactions entre adultes et adolescents polyhandicapés, nous avons mis en évidence l'importance de cette répétition qui permet à l'adulte d'établir et de maintenir la communication. En effet, les énoncés des adolescents sont très souvent défaillants sur les plans linguistique et/ou pragmatique. Si l'adulte ne montre pas à l'adolescent qu'il comprend que celui-ci est susceptible de vouloir exprimer quelque chose, l'interaction s'interrompt. Et nous avons observé que la stratégie la plus sûre (et la plus utilisée) pour signifier à l'adolescent la volonté de l'adulte de maintenir l'interaction verbale est justement de répéter l'élocution de l'adolescent, quel que soit son degré d'ambiguité (Bocéréan \& Musiol, 2009).

Notre objectif global est d'observer si le comportement communicatif (linguistique et pragmatique) des adultes diffère selon leur interlocuteur et, le cas échéant de quelle façon. Dans cet article, nous nous intéressons plus finement au type et à la fonction des répétitions dans chacun des deux corpus afin de préciser les différences dans l'emploi de cette stratégie par l'adulte face à un enfant typique ou face à un adolescent polyhandicapé. Plus précisément, nous faisons l'hypothèse que l'étude des types et fonctions des répétitions que l'adulte utilise selon qu'il interagit avec un enfant au développement typique ou un adolescent polyhandicapé est révélateur des représentations qu'a l'adulte des compétences interactionnelles de son interlocuteur. En comparant les deux corpus, nous observerons comment une même stratégie langagière, en l'occurrence la répétition, est déployée différemment par l'adulte face des 
interlocuteurs inégaux sur le plan de l'efficience linguistique et conversationnelle. Nous n'affirmons pas que l'adulte le fait consciemment mais le résultat est que ces différences observées engendrent des effets communicationnels qui diffèrent en fonction de l'interlocuteur ou plutôt en fonction de la représentation que l'adulte se fait de son interlocuteur.

Cette étude s'inscrit dans un objectif plus large, à savoir améliorer la communication avec des adolescents polyhandicapés, en mettant en évidence le comportement de l'adulte interlocuteur révélateur de représentations précises. Nous montrons que les adultes prêtent aux adolescents polyhandicapés des compétences communicationnelles et langagières inférieures à celles qu'ils attribuent aux jeunes enfants âgés de trois à cinq ans. Le comportement langagier des adultes vis-à-vis des adolescents est d'un niveau de complexité bien moindre que celui vis-à-vis de jeunes enfants. Pourtant, si les soignants considéraient les adolescents polyhandicapés comme des interlocuteurs à part entière et susceptibles d'apprentissage, ils pourraient contribuer à stimuler (ou tout au moins à considérer) l'efficience cognitive des adolescents.

Nous abordons ces analyses d'un point de vue psycholinguistique, par une double approche pragmatique et linguistique. En effet, ces deux approches sont complémentaires en ce sens où l'approche pragmatique met à jour des propriétés discursives communicationnelles et l'approche linguistique permet une analyse fine des énoncés des interlocuteurs, plus spécifiquement des constructions syntaxiques de l'adulte qui servent d'étayage aux verbalisations de l'enfant ou de l'adolescent polyhandicapé.

L'approche pragmatique étudie les conditions de possibilité de l'usage du langage. En ce sens, l'étude des interactions verbales recueillies de façon la plus écologique possible (c'est-à-dire des interactions les moins contraintes possibles, comme des conversations casuelles) permet d'aborder les caractéristiques du comportement des interlocuteurs. Nous basons notre approche sur la théorie des actes de langage (Searle, 1969/1972 ; Searle \& Vanderveken, 1985 ; Vanderveken \& Susumo, 2002) et sur une théorie de l'articulation et de la structure du discours (Roulet, Auchlin, Moeschler, Rubattel \& Schelling, 1985). Les formes d'interaction produites (intentionnellement ou non) par les interlocuteurs au cours d'un échange sont révélatrices de la compréhension et de l'analyse que fait chaque locuteur du déroulement de l'échange. Pour cette raison, notre unité d'analyse est l'échange conversationnel (ou transaction) et non pas l'énoncé. Ainsi, le principe de l'enchaînement conversationnel (Trognon \& Brassac, 1992) postule la production d'un premier énoncé E1 effectué par le locuteur L1, la production de E2 par L2 qui révèle la façon dont L2 interprète E1 et enfin la production de E3 par L1 qui valide, refuse ou module l'interprétation en actes par L2 de E1. Ce mécanisme débouche sur le phénomène d'intercompréhension car les interlocuteurs ont construit un sens commun. Ainsi, notre approche permet de dévoiler la construction dynamique conjointe du sens en interaction.

La répétition dans la conversation a souvent été étudiée par les auteurs, que ce soit au sein d'interactions verbales entre adultes (Clark \& Wasow, 1998; Curl, Local \& Walker 2006 ; Rieger, 2003 ; Schegloff, Jefferson \& Sachs, 1977) ou dans le cadre de l'étude du développement du langage (Bernicot, SalazarOrvig \& Veneziano, 2006; Chouinard \& Clark 2003 ; Clark et Chouinard, 2000). Les interactions verbales entre un adulte et un enfant souffrant de pathologie, comme la dysphasie ont également donné lieu à des recherches plus spécifiques (De Weck, 2006; Keith, Welsh, Camarata, Butkovsky, \& Camarata, 1995; Vigil, Hodges, \& Kle, 2005; Yont, Hewitt, \& Miccio, 2002). Les auteurs reconnaissent principalement deux fonctions à la répétition par l'adulte de l'énoncé de l'enfant (atteint ou non d'une pathologie). D'une manière générale, la première est de signaler à l'enfant s'il y a ou non partage de signification. Ainsi, en constituant le tour de parole suivant, la répétition permet de maintenir l'échange, de redonner la parole à son interlocuteur tout en l'informant que l'on a compris et accepté l'énoncé répété (Bernicot, Salazar-Orvig \& Veneziano, 2006 ; Clark, 2006). Autrement dit, le processus de répétition assure le lien thématique entre les actes de langage dans l'alternance des tours de parole et inscrit ainsi l'interlocuteur dans la séquence dialogique. Elle participe à la création et au maintien d'un espace intersubjectif (De Weck, 2006; Salazar-Orvig, 2000). L'énoncé ainsi partagé devient partie intégrante du «common ground» (Clark 1996). Lorsque les interlocuteurs n'ont pas le même degré de 
maîtrise de la langue, dans le cas d'interactions entre un adulte et un jeune enfant ou un enfant atteint d'une pathologie du langage, une seconde fonction s'ajoute à la première, celle d'étayage linguistique.

Certains auteurs ont détaillé plus précisément les fonctions des répétitions. Ainsi Bernicot, SalazarOrvig et Veneziano (2006) distinguent quatre fonctions : une fonction "texte» qui permet aux interlocuteurs de se mettre d'accord sur la forme des énoncés, ce qui implique leur correction phonologique, syntaxique, sémantique ou leur interprétation ; une fonction " états mentaux » qui permet le maintien du dialogue sous la forme d'une confirmation, d'un acquiescement, d'un refus, etc.; une fonction "assertive » qui précise la description du monde exprimée par les énoncés ; une fonction « directive » où la demande correspond à une tentative de faire faire quelque chose à son interlocuteur (information, action ou reformulation). Perrin, Deshaies et Paradis (2003) se basent quant à eux sur le modèle d'analyse du discours développé par Roulet (Roulet et al., 1985) pour différencier les fonctions pragmatiques de la répétition en tant que marqueurs de la structure du discours. La première fonction est appelée la fonction «de prise en compte» (« taking into account function»). Les auteurs postulent que toutes les répétitions ont cette fonction de base, qui consiste à indiquer que l'énoncé de l'interlocuteur a été entendu et interprété. Elle est nécessairement associée à l'une des trois autres fonctions : « demande de confirmation» (« confirmation request function»), « réponse positive», («positive reply function ») ou « réponse négative » (« negative reply function »). La répétition ayant pour fonction une demande de confirmation signale un problème relatif à l'énoncé de l'interlocuteur. La fonction de réponse positive exprime un accord de la part de celui qui répète, comme dans le cas de la fonction de prise en compte, mais cet interlocuteur assume en plus la responsabilité de l'énoncé. En reproduisant l'énoncé du locuteur, il ne lui fait pas seulement part qu'il l'a bien entendu et interprété, mais lui indique en plus qu'il fait sien de son énoncé. Enfin, lors d'une répétition ayant une fonction de réponse négative, l'interlocuteur manifeste son désaccord avec cet énoncé. Nous pouvons préciser que cette fonction n'apparaît pas dans nos deux corpus; en effet, il semble que les adultes, lorsqu'ils s'adressent à un enfant ou un adolescent polyhandicapé ne montrent aucun désaccord avec eux. Nous nous baserons sur ces diverses approches pour proposer un modèle de codage de la fonction pragmatique des répétitions observées dans nos deux corpus.

L'approche linguistique interactionnelle, quant à elle, prend sa source dans les recherches en acquisition du langage portant sur le langage adressé à l'enfant. Sur fond de controverses sur le rôle de l'input dans l'appropriation du langage par l'enfant (Lieven \& Pine, 1993), deux démarches s'inscrivent dans ce paradigme conversationnel :

- les études quantitatives, qui établissent des corrélations entre le langage adressé à l'enfant et l'évolution du langage de l'enfant et qui mettent mis en évidence des stratégies d'ajustements intuitives de l'adulte. Ces études portent pour l'essentiel sur l'émergence lexicale et grammaticale chez les enfants de moins de 3 ans (Gallaway \& Richard, 1994 ; Snow, 1995 pour une synthèse);

- les études dialogiques, s'appuyant entre autres sur les travaux précurseurs de Bruner (1983).

Dans cette dernière perspective, les recherches ont tenté de dépasser les limites d'une analyse strictement statistique en analysant le déroulement des dialogues et leurs liens discursifs au-delà des seules paires adjacentes (par exemple Hudelot, 1987 pour le français). Mais une implication de l'enfant dans le dialogue est aussi le moyen de s'approprier les constructions de la langue, ce que montre par exemple Veneziano (2000) qui combine la dimension pragmatique à une perspective socioconstructiviste pour étudier le langage de très jeunes enfants. Plusieurs autres recherches sur des enfants francophones entre 3 et 6 ans ont cherché à valider le rôle des interactions dans l'apprentissage, et plus précisément à démontrer que l'adulte en position d'étayeur s'adapte aux capacités langagières potentielles de l'enfant pour qu'elles deviennent source d'appropriation pragma-linguistique, pour que s'établissent progressivement l'organisation de ressources langagières. Par exemple Canut (2006, 2009), Canut et Vertalier (2005) et Karnoouh-Vertalier (1998) ont cherché à vérifier le principe d'une acquisition progressive s'accompagnant d'un degré de complexité syntaxique croissant en lien avec le contenu syntaxique des répétitions et reformulations de l'adulte. 
En confrontant les formulations linguistiques et les caractéristiques pragmatiques des échanges entre un adulte et un adolescent polyhandicapé d'une part, et un adulte et un jeune enfant d'autre part, nous serons à même d'observer leurs différences d'intentions, de comportement, de représentations, de stratégies linguistiques.

\section{Participants et procédure}

Le corpus polyhandicapé a été recueilli dans une Institution Spécialisée en France qui accueille une soixantaine d'enfants et adolescents polyhandicapés. La première phase de la recherche a consisté en une observation participante de plusieurs mois, au cours de laquelle ont été sélectionnés les adolescents susceptibles de communiquer verbalement, ne serait-ce qu'a minima. Six adolescents, deux garçons et quatre filles, (soit 10\% des patients de l'Institution) se sont avérés susceptibles de contribuer de l'une ou l'autre manière au processus d'accomplissement de l'intercompréhension dans l'échange conversationnel. Le tableau 1 réunit les principales informations concernant ces adolescents. Leur degré de déficience mentale est très élevé ; tous se déplacent en fauteuil roulant et seulement deux d'entre eux sont capables de faire quelques pas avec un déambulateur. Leur degré de compréhension verbale est variable mais tous souffrent de sévères difficultés à s'exprimer verbalement et non verbalement à cause de leurs lourdes paralysies. Ainsi, il n'est pas possible de tenir compte de la prosodie car, dans la plupart des cas, les adolescents polyhandicapés ne maîtrisent pas leur voix et s'expriment en criant.

\begin{tabular}{|c|c|c|}
\hline $\begin{array}{l}\text { adolescent } \\
\text { polyhandicapé }\end{array}$ & âge mental & diagnostic (nature des séquelles) \\
\hline Laure, 15 ans & 3 ans-3; 6 ans & $\begin{array}{l}\text { encéphalopathie de prématurité (déficit intellectuel ; } \\
\text { épilepsie ; paralysie des membres inférieurs) }\end{array}$ \\
\hline Lola, 17 ans & $3 ; 6$ ans -4 ans & $\begin{array}{l}\text { conséquences d'une naissance prématurée (déficit } \\
\text { intellectuel; épilepsie; infirmité motrice; insuffisance } \\
\text { cardiaque) }\end{array}$ \\
\hline Elise, 15 ans & 4-5 ans & $\begin{array}{l}\text { conséquences d'une souffrance fœetale (déficit intellectuel ; } \\
\text { paralysie des membres inférieurs) }\end{array}$ \\
\hline Alban, 17 ans & 3 ans- $3 ; 6$ ans & $\begin{array}{l}\text { leucodystrophie congénitale (déficit intellectuel ; épilepsie ; } \\
\text { paraplégie ; ne se nourrit pas par voie orale) }\end{array}$ \\
\hline Bernard, 17 ans & $4 ; 6$ ans -5 ans & $\begin{array}{l}\text { conséquence d'un traumatisme crânien avec perte de } \\
\text { conscience; accident de la voie publique à l'âge de } 6 \text { ans } \\
\text { (déficit intellectuel ; épilepsie; tétraplégie) }\end{array}$ \\
\hline Cathy, 16 ans & $\begin{array}{l}4 ; 9 \text { ans- } 5 ; 3 \\
\text { mois }\end{array}$ & $\begin{array}{l}\text { conséquences d'une souffrance fotale (déficit intellectuel ; } \\
\text { épilepsie ; tétraplégie épargnant le bras droit) }\end{array}$ \\
\hline
\end{tabular}

Tableau 1. Diagnostics et séquelles des adolescents polyhandicapés

Chaque patient retenu est enregistré à plusieurs reprises sur support vidéo alors qu'il interagit avec une ou plusieurs personnes soignantes. Les échanges verbaux ont lieu lors d'activités non dirigées, lors de moments durant lesquels les adolescents discutent avec les personnes les accompagnant, en attendant le repas, des activités précises, des soins, etc. Ces entretiens sont composés d'un certain nombre de « transactions » c'est-à-dire d'échanges plus ou moins complexes structurés sur la base de thématiques congruentes. Ce corpus contient 82 transactions correspondant à 1961 tours de parole. L'âge mental moyen, estimé par les tests verbaux et non verbaux du Terman-Merrill et du WPPSI-R, des six adolescents est de quatre ans et un mois. 
Pour constituer le corpus témoin, nous avons décidé de nous baser sur l'âge mental. C'était une donnée dont nous disposions pour les adolescents polyhandicapés (qui sont testés régulièrement) et cela n'avait évidemment aucun sens d'apparier les adolescents polyhandicapés avec d'autres adolescents de même âge. Les interlocuteurs des adolescents polyhandicapés sont des personnels soignants (aides-soignants, infirmières, psychologues stagiaires), c'est-à-dire des personnes qui côtoient journellement les adolescents dans l'institution. En ce qui concerne les interlocuteurs des jeunes enfants du corpus témoin, il n'était pas question que ce soit des parents ou des enseignants; en effet, les interactions verbales entre un parent et son enfant ou un enseignant et son élève sont beaucoup trop spécifiques pour que l'on puisse les considérer comme étant comparables aux interactions entre les adolescents et le personnel de l'institution. Le corpus témoin a donc été recueilli dans un centre de vacances ouvert à des enfants âgés de trois à six ans. Après avoir passé plusieurs jours dans le centre de vacances pour se faire connaître des enfants, deux psychologues stagiaires ont filmé les interactions verbales que l'une d'entre elles ou un adulte animateur avait avec certains enfants lors d'activités libres, le plus souvent à l'extérieur avant ou après les repas. Ni les psychologues stagiaires, ni les animateurs du centre de vacances n'étaient au courant des objectifs de la recherche. Cinq petits garçons et trois petites filles ont ainsi participé à l'étude. Leur âge moyen est de quatre ans et cinq mois.

\section{Codage des interactions}

Toutes les interactions verbales ont été filmées. Deux chercheurs ont chacun retranscrit l'ensemble des corpus et ont comparé ensuite leurs retranscriptions. Les expressions incompréhensibles et les approximations ont été retranscrites phonétiquement. Les désaccords ont été résolus à l'aide d'une tierce personne. Les répétitions ont été codées en prenant comme unité d'analyse le tour de parole. Ainsi, les hétéro répétitions correspondent à la phrase initiale, répétée par l'interlocuteur dès le tour de parole suivant. Les auto répétitions sont codées en tant que telles lorsque, au tour de parole $n+2$, le locuteur reprend en partie ou en totalité la phrase qu'il a énoncée lors du tour de parole $n$.

On observe tout d'abord qu'il y a deux fois plus de répétitions dans le corpus polyhandicapé (6.94\% des tours de parole) que dans le corpus témoin (3.37\%). Mais, si les corpus se différencient quant au nombre de répétitions, ils sont semblables quant au type de répétitions. En effet, dans les deux corpus, les répétitions sont en grande majorité des hétéro répétitions $(96 \%$ dans le corpus polyhandicapé ainsi que dans le corpus témoin). Ce sont principalement les adultes qui répètent les propos de l'enfant ou de l'adolescent(e) : 72.8\% des hétéro-répétitions sont le fait d'adulte ( $92.4 \%$ dans le corpus témoin).

Pour analyser plus finement les types de répétition, nous avons utilisé le codage de Bernicot, SalazarOrvig et Veneziano (2006) qui différencie d'une part les répétitions complètes (avec ou sans ajout) des répétitions partielles (avec ou sans ajout) et d'autre part, les auto et hétéro répétitions. Dans le tableau 2 nous présentons un exemple de chacun des huit types possibles de répétition. 


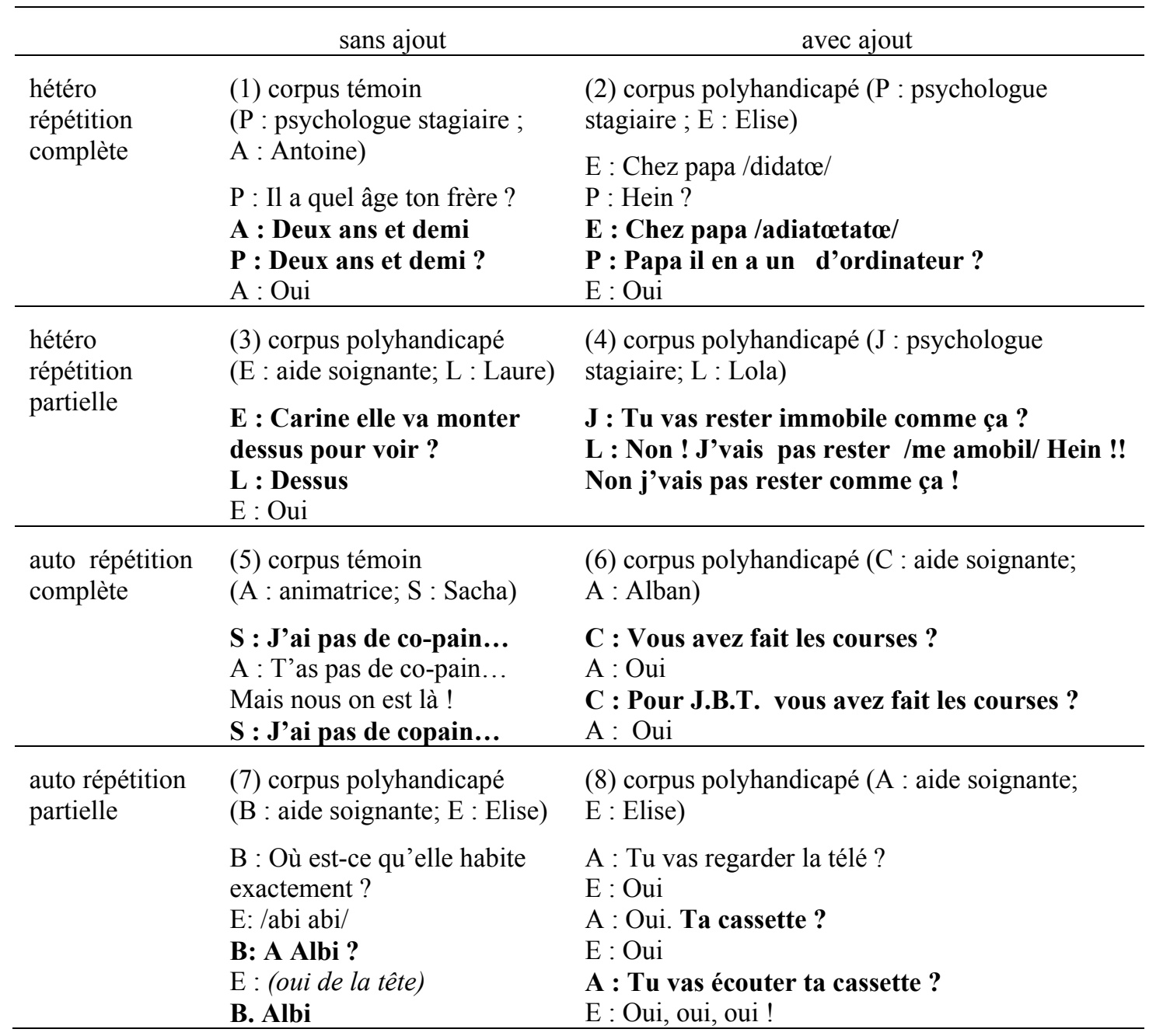

Tableau 2. Exemple des huit types de répétition

\section{Résultats}

Nous avons comptabilisé et analysé les différents types de répétitions relevés dans les deux corpus (cf. tableau 3). 


\begin{tabular}{|c|c|c|c|c|}
\hline \multirow[b]{2}{*}{$\mathrm{N}(\%)$} & \multicolumn{2}{|c|}{ corpus polyhandicapé } & \multicolumn{2}{|c|}{ corpus témoin } \\
\hline & $\begin{array}{l}\text { répétition par } \\
\text { l'adulte }\end{array}$ & $\begin{array}{l}\text { répétition par } \\
\text { l'adolescent }\end{array}$ & $\begin{array}{l}\text { répétition par } \\
\text { l'adulte }\end{array}$ & $\begin{array}{l}\text { répétition par } \\
\text { l'adolescent }\end{array}$ \\
\hline H R complète avec ajout & 46 & 1 & 14 & l \\
\hline H R complète sans ajout & 39 & 16 & 28 & 2 \\
\hline H R partielle avec ajout & 2 & 6 & 7 & I \\
\hline H R partielle sans ajout & 12 & 8 & 14 & / \\
\hline A R complète avec ajout & 3 & / & I & / \\
\hline A R complète sans ajout & 1 & 1 & 1 & 2 \\
\hline A R partielle avec ajout & / & / & / & / \\
\hline A R partielle sans ajout & 1 & 1 & 1 & 1 \\
\hline $\mathrm{N}$ & 104 & 32 & 63 & 5 \\
\hline
\end{tabular}

Tableau 3. Nombre et pourcentages de répétitions par type et par corpus ( $\mathrm{H} \mathrm{R} »$ : hétéro répétition; « $\mathrm{A}$ $\mathrm{R} »$ : auto répétition)

Deux observations principales se dégagent de cette classification. La première est que les adolescents polyhandicapés répètent beaucoup plus souvent les énoncés de l'adulte que les jeunes enfants $\left(\mathrm{X}^{2}(1\right.$, $195)=13.3, \mathrm{p}=.001)$. La seconde observation concerne les hétéro répétitions : nous avons noté que l'adulte peut répéter l'énoncé de son interlocuteur soit en ajoutant quelque chose ( $c f$. . (2) et (4)), soit en le reprenant tel quel ( $c f$. (1) et (3)). La différence concernant les hétéro répétitions dans les deux corpus se situe à ce niveau : l'adulte enrichit l'énoncé de son interlocuteur dans $34.5 \%$ des cas lorsqu'il s'adresse à un adolescent polyhandicapé alors qu'il le fait dans $46 \%$ des cas lorsqu'il s'agit d'un jeune enfant.

Nous venons de constater que l'adulte complète plus souvent l'énoncé de son interlocuteur lorsque celui-ci est un jeune enfant plutôt qu'un adolescent polyhandicapé. La différence ne se situe pas seulement à ce niveau quantitatif; nous analysons maintenant le type d'ajout de la part de l'adulte selon son interlocuteur.

\subsection{Caractéristiques linguistiques des hétéro répétitions avec ajout de la part de l'adulte}

Nous avons déterminé la forme linguistique des énoncés de l'adulte, des énoncés des enfants ou des adolescents en les catégorisant syntaxiquement de la façon suivante (L1 et L2 sont les deux locuteurs) :

- les constructions incomplètes :

Le locuteur produit un morphème isolé (MI) ; il s'agit d'un élément isolé (nom, verbe, adjectif...) dépourvu de déterminant ou de préposition et de noyau verbal dans l'énoncé considéré.

Exemple : $\quad$ L1 :/mine/

L2 : Che-mi-née

Le locuteur produit un syntagme isolé (SI) ; il s'agit d'un syntagme nominal, adjectival, prépositionnel dépourvu de noyau verbal dans l'énoncé considéré.

Exemple: $\quad$ L1 : Un dessin de quoi ?

L2 : De cochon 
- les constructions complètes :

Le locuteur produit une ou plusieurs constructions simples juxtaposées ou coordonnées (CS). Il y a présence d'un noyau verbal et absence de subordination.

Exemple : $\quad$ L1 : Ils les tuent

L2 : Ils les tuent? Les machines tuent les robots? Hein?

Le locuteur produit une construction complexe (CC); les complexités syntaxiques correspondent globalement aux subordonnées (complétives, relatives, circonstancielles).

Exemple : $\quad$ L1 : Là, elle qui conduit, là

L2: Ah... Celle qui conduit, tu l'aimes bien?

Un énoncé peut comporter plusieurs types de constructions. Pour l'adulte, nous avons différencié les constructions qui concernent la répétition même et les constructions qui concernent une éventuelle extension de cette répétition. L' «extension» peut prendre différentes formes : une question, une demande de clarification, une explication supplémentaire, un encouragement, etc. D'un point de vue strictement linguistique, il s'agit d'un prolongement, d'un ajout d'éléments nouveaux (CS, CC ou syntagme prépositionnel ou nominal). C'est le cas des deux exemples précédents : « les machines tuent les robots » est une extension de la répétition « ils les tuent »; « tu l'aimes bien » est une extension de la répétition « celle qui conduit».

Nous nous sommes d'abord intéressés au type de verbalisation produit par les enfants et les adolescents et répétés par les adultes. Les résultats reportés dans le tableau 4 indiquent que la composition linguistique des énoncés des enfants et des adolescents est très différente : les adolescents polyhandicapés verbalisent essentiellement des morphèmes ou syntagmes isolés $(85.41 \%$ au total) alors que les enfants produisent une majorité de constructions complètes simples ou complexes $(80.95 \%$ au total).

\begin{tabular}{rcc}
\hline type de construction (\%) & enfants & adolescents polyhandicapés \\
\hline Morphème Isolé (MI) & $/$ & 43.8 \\
\hline Syntagme Isolé (SI) & 19 & 41.7 \\
\hline Construction Simple (CS) & 71.4 & 12.5 \\
\hline Construction Complexe (CC) & 9.5 & 2.1 \\
\hline nombre d'énoncés & 21 & 48 \\
\hline
\end{tabular}

Tableau 4. Caractéristiques linguistiques des énoncés des enfants et des adolescents ayant impliqué une hétéro répétition avec ajout

On peut faire l'hypothèse que les différences entre les types de verbalisation observés chez les enfants et les adolescents sont susceptibles d'influencer les adultes lorsqu'ils répètent les énoncés de leur interlocuteur. Plus précisément, l'usage par les enfants de constructions ayant un certain degré de complétude et de complexité a pu (intuitivement) amener l'adulte à proposer des allongements. Pour tenter de répondre à cette hypothèse, nous avons observé les caractéristiques linguistiques des hétéro répétitions de la part des adultes ( $c f$. tableau 5). 


\begin{tabular}{rcc}
\hline & $\begin{array}{c}\text { répétitions des énoncés des } \\
\text { enfants }\end{array}$ & $\begin{array}{c}\text { répétitions des énoncés des } \\
\text { adolescents }\end{array}$ \\
\hline $\mathrm{N}(\%)$ & $/$ & 4 \\
$\mathrm{MI}$ & 1 & 12 \\
$\mathrm{SI}$ & 1 & 12 \\
$\mathrm{CS}$ & 2 & 2 \\
$\mathrm{CC}$ & $/$ & 6 \\
$\mathrm{MI}+$ extension & 5 & 6 \\
$\mathrm{SI}+$ extension & 10 & 5 \\
$\mathrm{CS}+$ extension & 2 & 1 \\
$\mathrm{CC}+$ extension & 21 & 48 \\
\hline nombre d'énoncés & & \\
\hline
\end{tabular}

Tableau 5. Caractéristiques linguistiques des hétéro répétitions de la part des adultes selon leur interlocuteur

On observe des différences assez nettes entre les deux corpus : $81 \%$ des répétitions des énoncés des enfants comportent une extension; à l'inverse, les répétitions des énoncés des adolescents sont majoritairement sans extension (62.5\%). Parmi ces répétitions sans extension, le pourcentage concerne pour moitié des constructions incomplètes (33.3\%).

De plus, si on se focalise sur les extensions produites par l'adulte lors des répétitions des énoncés des enfants ou des adolescents, on observe une autre différence intéressante : sur les 17 répétitions comportant des extensions des énoncés des enfants, $35.3 \%$ sont des constructions complexes alors que sur les 18 répétitions comportant des extensions des énoncés des adolescents polyhandicapés, seulement $11.1 \%$ sont des constructions complexes.

Les résultats tendent donc à montrer qu'avec les enfants, l'adulte propose davantage d'extensions (il va au-delà de la simple répétition de ce que dit l'enfant) et qu'il complexifie davantage ses énoncés (du point de vue de la forme syntaxique) qu'avec les adolescents polyhandicapés. On peut supposer que l'interaction dépend de ce que l'adulte perçoit comme développement possible : pour l'enfant qui serait potentiellement capable de capter des éléments dans le langage qui lui est adressé, l'adulte se place dans une zone proche de développement (qui excède légèrement les capacités langagières de l'enfant). Pour les adolescents, l'adulte serait moins dans une logique d'apprentissage et chercherait davantage à un maintien de la communication par un jeu de répétitions simples.

Cette interprétation est confirmée par une analyse des fonctions pragmatiques des répétitions de la part de l'adulte.

\subsection{Fonctions pragmatiques des répétitions}

Nous nous sommes inspirés des travaux décrits en introduction pour définir trois fonctions qui correspondent plus précisément à ce que nous avons observé lors de l'étude des différents types de répétition relevés dans nos corpus.

Conformément à Perrin, Deshaies et Paradis (2003), nous considérons que la fonction de base de maintien de l'échange («taking into account function») est inhérente à toute hétéro répétition. Ce maintien de l'échange peut néanmoins prendre plusieurs formes dialogiques qui reflètent des fonctions différentes : «compréhension mutuelle », «enrichissement linguistique » et «enrichissement pragmatique $»$. 
- La fonction de compréhension mutuelle ( $c f$. tableau 6, exemples (9) et (10))

L'adulte reprend partiellement ou complètement l'énoncé de l'enfant ou de l'adolescent en ne changeant rien ni à la forme propositionnelle ni au sens linguistique de cet énoncé ; dans la classification utilisée précédemment, ce type de répétition correspond aux répétitions partielles et complètes sans ajout. Il a pour fonction de signifier à l'enfant ou à l'adolescent que l'adulte reprend l'énoncé à son compte (même s'il n'est répété que partiellement), qu'il le fait sien, qu'il le reconnaît en tant que partie intégrante du commun ground (Clark, 1996). Cette fonction correspond en partie à la " positive reply function » et à la « confirmation request function» de Perrin et al. (2003).

- La fonction d'enrichissement linguistique ( $c f$. tableau 6, exemples (11) et (12))

L'adulte remarque une ambiguité linguistique dans l'énoncé de l'enfant; il reprend cet énoncé en proposant à son interlocuteur une formulation adéquate. L'ambiguité peut être d'ordre phonologique, syntaxique ou grammatical. Ce type de répétition correspond généralement à une répétition partielle ou complète avec ajout. Elle a un rôle d'étayage linguistique. Cette fonction correspond à la fonction « texte» de Bernicot, Salazar-Orvig et Veneziano (2006).

- La fonction d'enrichissement pragmatique ( $c f$. tableau 6, exemples (13) à (18))

L'adulte reprend l'énoncé de l'enfant en l'enrichissant sur le plan pragmatique; il peut le faire de trois façons : 1) en émettant une hypothèse sur l'intention de sens (il fait une inférence), 2) en signifiant le partage d'états mentaux (il montre son approbation, il complimente, etc.), 3) il relance le dialogue en poursuivant sur le même thème. Ce type de répétition correspond aux répétitions partielles et complètes avec ajout. Cette fonction est incluse dans les fonctions « états mentaux », « assertif », voire « directif » de Bernicot, Salazar-Orvig et Veneziano (2006). Elle a nécessairement pour caractéristiques celles de la fonction de compréhension mutuelle; elle signifie en plus à l'enfant ou à l'adolescent que l'adulte a compris et interprété son énoncé non seulement au niveau linguistique mais surtout au niveau de son intention de sens. En enrichissant d'une façon ou d'une autre l'énoncé de l'enfant ou de l'adolescent, l'adulte lui montre ainsi qu'il le considère comme un interlocuteur accompli dont la conversation est digne d'intérêt. 


\begin{tabular}{|c|c|c|}
\hline $\begin{array}{l}\text { fonctions des } \\
\text { répétitions }\end{array}$ & corpus témoin & corpus polyhandicapé \\
\hline \multirow{2}{*}{$\begin{array}{l}\text { compréhension } \\
\text { mutuelle }\end{array}$} & (9) P : animateur ; S: Sacha & (10) A : aide soignante; E : Elise \\
\hline & $\begin{array}{l}\text { P : T'as eu mal un p'tit peu? } \\
\text { S : Ouais, j'avais du sang ! } \\
\text { P : T'avais du sang? } \\
\text { S : (acquiesce de la tête) }\end{array}$ & $\begin{array}{l}\text { A : Comment elles s'appellent? } \\
\text { E : Celine et Charlotte } \\
\text { A : Charlotte and Celine (?) } \\
\text { E : Oui }\end{array}$ \\
\hline \multirow{2}{*}{$\begin{array}{l}\text { enrichissement } \\
\text { linguistique }\end{array}$} & (11) A : animatrice; $\mathrm{M}$ : Marie & (12) $\mathrm{P}$ : aide soignant ; E : Elise \\
\hline & $\begin{array}{l}\text { A : Vous avez fait quoi toutes les } \\
\text { deux (?) Vous êtes allée un p'tit peu } \\
\text { vous promener? } \\
\text { M : MJC } \\
\text { A : Ah vous êtes allées à la MJC } \\
\text { M : Oui }\end{array}$ & $\begin{array}{l}\text { E : [a ma e juillet, a ma e juillet] } \\
\text { P : Le mois de juillet } \\
\text { E : Oui }\end{array}$ \\
\hline \multirow{2}{*}{$\begin{array}{l}\text { enrichissement } \\
\text { pragmatique avec } \\
\text { inférence }\end{array}$} & (13) $\mathrm{P}$ : animateur ; S : Sacha & (14) M : aide soignant; B : Bernard \\
\hline & $\begin{array}{l}S: \text { Après, ça s'ra les grandes } \\
\text { vacances de mer ! } \\
P \text { : « Les grandes vacances de } \\
\text { mer »... Ton papa, il va t'emmener } \\
\text { en vacances à la mer... } \\
S \text { : Ouais }\end{array}$ & $\begin{array}{l}\text { B : Moi Popeye } \\
\text { M : Toi Popeye, t'es fort? T'es } \\
\text { costaud? } \\
\text { B : Oui (fait le geste de montrer ses } \\
\text { muscles) }\end{array}$ \\
\hline \multirow{2}{*}{$\begin{array}{l}\text { enrichissement } \\
\text { pragmatique avec } \\
\text { partage d'états mentaux }\end{array}$} & (15) $\mathrm{P}$ : animatrice ; $\mathrm{C}$ : Corentin & (16) P : aide soignante; L : Lola \\
\hline & $\begin{array}{l}\text { C : Et ben moi aussi j'ai quatre } \\
\text { ans. Moi aussi j'ai quatre ans ! Moi } \\
\text { j'ai quatre ans. } \\
\mathbf{P}: \text { Hop là ! A tes souhaits ! T'as } \\
\text { quatre ans? T'as aussi des frères et } \\
\text { sœurs? } \\
\mathrm{C}:(\text { acquiesce de la tête) }\end{array}$ & $\begin{array}{l}\text { L : Regarde (en montrant ses } \\
\text { doigts) P : C'est quoi alors? } \\
\mathrm{L}: \text { C'est du ... } \\
\mathrm{P}: \text { Du vernis? } \\
\text { L : Non des paillettes } \\
\text { P : Des paillettes !... wouah !! } \\
\text { L : Oui des paillettes }\end{array}$ \\
\hline \multirow{2}{*}{$\begin{array}{l}\text { enrichissement } \\
\text { pragmatique avec } \\
\text { relance thématique }\end{array}$} & (17) A : animatrice; $\mathrm{N}$ : Nicolas & (18) B : aide soignante; E : Elise \\
\hline & $\begin{array}{l}\text { A : T'as pas un p'tit frère ici toi ? } \\
\text { N : Non j'ai un grand frère } \\
\text { A : Non t'as un grand frère. Alors } \\
\text { c'est lequel ton grand frère? }\end{array}$ & $\begin{array}{l}\text { B : Il est où maintenant? } \\
\text { E : A Toul } \\
\text { B : A Toul et il s'occupe toujours } \\
\text { des hélicoptères? }\end{array}$ \\
\hline
\end{tabular}

Tableau 6 : Exemples de chacune des fonctions pragmatiques de la répétition

Nous avons codé toutes les hétéro répétitions des deux corpus ( $c f$. tableau 7).

\begin{tabular}{rcccc}
\hline & $\begin{array}{c}\text { fonction de } \\
\text { compréhension } \\
\text { mutuelle }\end{array}$ & $\begin{array}{c}\text { fonction } \\
\text { d'enrichissement } \\
\text { linguistique }\end{array}$ & $\begin{array}{c}\text { fonction } \\
\text { d'enrichissement } \\
\text { pragmatique }\end{array}$ & total \\
\hline corpus polyhandicapé & $48(48.5)$ & $43(43.4)$ & $8(8.1)$ & 99 \\
corpus témoin & $39(61.9)$ & $3(4.8)$ & $21(33.3)$ & 63 \\
\hline
\end{tabular}

Tableau 7. Nombre et pourcentage de répétitions selon leur fonction et selon le corpus 
Les fonctions des répétitions ne sont pas identiques dans les deux corpus : la différence la plus importante se situe au niveau des fonctions d'enrichissement linguistique et pragmatique $\left(X^{2}(1,75)=\right.$ $35.5, \mathrm{p}=.001$ ). Alors qu'un tiers des répétitions dans le corpus témoin ont pour fonction l'enrichissement pragmatique, seulement $8 \%$ des répétitions ont cette fonction dans le corpus polyhandicapé. Les adultes, lorsqu'ils ne répètent pas à l'identique l'énoncé d'un adolescent polyhandicapé, le font principalement dans un but d'étayage linguistique. On observe également que : 1) les corrections linguistiques sont principalement des corrections phonétiques, 2) les adultes répètent parfois à l'identique l'énoncé de l'adolescent même si celui-ci n'est pas adéquat linguistiquement ( $c f$. exemple 19)

(19) : corpus polyhandicapé (P : psychologue; E : Elise)

$$
\begin{aligned}
& \mathrm{P}: \text { Vous étiez trois? } \\
& \mathbf{E}: \text { Oui, nous trois filles } \\
& \mathbf{P}: \text { Trois filles } \\
& \mathrm{E}: \text { Oui }
\end{aligned}
$$

Le pourcentage de répétitions à fonction d'étayage linguistique n'est pas étonnant, compte tenu des difficultés d'expression verbale des adolescents polyhandicapés. On retrouve des résultats similaires dans les études portant sur des enfants dysphasiques. Les adultes, lorsqu'ils interagissent avec un jeune enfant, privilégient donc le versant pragmatique qui leur apparait d'importance moindre dans le cas d'interactions verbales avec des adolescents polyhandicapés.

\section{Conclusion}

L'objectif principal de cette étude était de comparer le comportement communicatif d'adultes face à des adolescents polyhandicapés ou face à des enfants au développement typique de même âge mental que les adolescents en nous basant sur le type et la fonction des répétitions utilisées par l'adulte en conversation. En effet, les caractéristiques discursives de l'adulte sont révélatrices de la représentation qu'il se fait des compétences interactionnelles de son interlocuteur. Ainsi, notre but était d'observer si les adultes interagissant verbalement avec des adolescents polyhandicapés monopolisent bien toutes les compétences possibles de leur interlocuteur comme ils le font lorsqu'ils sont face à un enfant au développement typique.

Nous avons abordé ce questionnement sous l'angle de l'analyse et de la comparaison des répétitions, fortement présentes dans les interactions verbales des deux corpus étudiés. Un résultat principal s'est dégagé de l'analyse globale des interactions verbales: dans les deux corpus, les répétitions sont majoritairement des hétéro répétitions : l'adulte répète l'énoncé des enfants et des adolescents; mais elles ne sont pas de même type dans les deux corpus : lorsqu'un adulte répète les propos d'un jeune enfant, il enrichit plus souvent sa répétition (hétéro répétition avec ajout) qu'il ne le fait lorsqu'il interagit avec un adolescent polyhandicapé. Nous avons alors centré nos analyses sur les formes et surtout sur les fonctions des répétitions par les adultes des énoncés des enfants et des adolescents. L'étude de la forme linguistique des énoncés produits par l'adulte en réaction à ceux produits par les enfants ou adolescents a montré que l'adulte propose d'avantage d'extensions et de complexifications aux enfants au développement typique qu'aux adolescents polyhandicapés. Nous avons complété cette analyse linguistique en étudiant la fonction des différents types d'hétéro répétition. La principale différence entre les deux corpus réside dans le fait que les répétitions par les adultes de l'énoncé d'un jeune enfant ont beaucoup plus souvent pour fonction l'enrichissement pragmatique que les répétitions des énoncés des adolescents polyhandicapés.

Ainsi, les deux approches (linguistique et pragmatique) se rejoignent en mettant en évidence un comportement langagier et communicationnel des adultes différent selon leur interlocuteur. L'adulte, lorsqu'il interagit avec un adolescent polyhandicapé, privilégie le maintien du dialogue: il use de répétitions pures (c'est-à-dire strictement identiques à l'énoncé répété) ou il corrige la prononciation phonétique de l'énoncé. Il ne propose pas d'énoncés plus complexes, qui seraient susceptibles de participer à une stratégie d'étayage linguistique. D'un point de vue de la structure des échanges, ce type de répétition fait que l'adulte redonne la parole à son interlocuteur mais sans s'impliquer d'avantage 
dans l'interaction. L'interprétation du comportement de l'adulte face à un interlocuteur polyhandicapé peut être double : d'une part, il semble que l'adulte ne se représente pas l'adolescent polyhandicapé comme susceptible de se trouver dans une position d'apprentissage linguistique; il prend ainsi acte de son handicap. Mais d'autre part, la stratégie de répétition à l'identique (ou corrigée phonétiquement) de l'énoncé de l'adolescent indique un comportement communicationnel extrêmement charitable de la part de l'adulte : l'adulte montre à son interlocuteur qu'il lui prête des compétences conversationnelles suffisantes pour une compréhension mutuelle et qu'il le laisse gérer lui-même la poursuite de l'échange. Néanmoins, par ce type de comportement, l'adulte ne favorise pas le développement possible langagier et communicationnel de l'adolescent. En effet, s'il est vrai que l'acquisition du langage dépend de multiples facteurs, s'il est vrai qu'une privation d'input peut sévèrement retarder l'acquisition, ou inversement que des éléments présentés fréquemment en input sont acquis plus rapidement, une quantité limitée de verbalisation d'éléments pertinents est souvent suffisante pour jouer un rôle catalytique (Nelson, Welsh, Camarata, Tjus \& Heimann, 2001) : l'enfant a la capacité de s'approprier très vite des éléments alors même qu'on ne les trouve que rarement dans le langage de l'adulte. Or, les évaluations psychologiques et psychométriques régulières des adolescents font état de capacités d'apprentissage, certes faibles, mais concrètes. D'un point de vue clinique, il nous semble important que les soignants considèrent que ces adolescents ont de réelles capacités cognitives (au risque de les surévaluer) plutôt que de les confiner dans un rôle d'interlocuteur passif dont les compétences seraient à jamais figées du fait de leur polyhandicap.

\section{Références bibliographiques}

Bernicot, J., Salazar-Orvig, A. \& Veneziano, E. (2006). Les reprises : dialogue, formes, fonctions et ontogenèse. La Linguistique, 42, 30-50.

Bocéréan, C. \& Musiol, M. (2009). Mutual understanding mechanism in verbal exchanges between carers and multiply-disabled young people: an interaction structure analysis. Pragmatics, 19 (2), $161-177$

Bruner, J. S. (1983). Le développement de l'enfant : savoir faire, savoir dire. Paris : P.U.F.

Canut, E. (2006). Apprentissage du langage oral et accès à l'écrit. Travailler avec un chercheur dans l'école. Amiens : CRDP d'Amiens / Scérén.

Canut, E. (2009). La syntaxe: un socle indispensable à l'apprentissage du langage. Les conceptions interactionnistes de la linguistique de l'acquisition à la lumière des approches contemporaines. L'exemple de parce que. In E. Canut, \& M. Vertalier (eds.), L'apprentissage du langage: une approche interactionnelle. Réflexions théoriques et pratiques de terrain. Paris : L'Harmattan, 69125.

Canut, E. \& Vertalier, M. (2005). Un fonctionnement-clé de l'acquisition du langage: essais et reformulations dans les interactions adulte-enfant. Colloque RRR : Répétition, Reprises et Reformulations. Quels usages dans les interactions verbales? Paris : LEAPLE, 1-2 avril.

Chouinard, M. M., \& Clark, E. V. (2003). Adult reformulations of child errors as negative evidence. Journal of child language, 30, 637-669.

Clark, E. V. (2006). La répétition et l'acquisition du langage. La Linguistique, 42, 67-79.

Clark, E. V., \& Chouinard, M. M. (2000). Enoncés enfantins et reformulations adultes dans l'acquisition du langage. Langage, 140, 9-23.

Clark, H. H. (1996). Using language. Cambridge: C.U.P.

Clark, H. H., \& Wasow, T. (1998). Repeating words in spontaneous speech. Cognitive psychology, 37, 21-242. 
Curl, T. S., Local J., \& Walker, G. (2006). Repetition and the prosody-pragmatics interface. Journal of pragmatics, $38(10), 1721-1751$.

De Weck, G. (2006). Les reprises dans les interactions adulte-enfant: comparaison d'enfants dysphasiques et tout-venant. La Linguistique, 42, 115-133.

Gallaway, C., \& Richards, B. (1994). Input and Interaction in Language Acquisition. Cambridge: Cambridge University Press.

Hudelot, C. (1987). Organisateurs discursifs du dialogue adulte-enfant : une esquisse tracée à partir de quelques dialogues (enfants de 5 à 6 ans). Modèles linguistiques, IX, 1, 33-51.

Karnoouh-Vertalier, M. (1998). Evolution du fonctionnement syntaxique et variantes énonciatives. Observations d'interactions langagières entre adulte et enfant au cours d'activités de narration. Langue Française, 118, 84-103.

Keith, E., N., Welsh, J., Camarata, S., M., Butkovsky, L., \& Camarata, M. (1995). Available input for language-impaired children and younger children of matched language levels. First Language, 15 (43), 1-17.

Lieven, E. V. M., \& Pine, J. M. (1993). Exposition et appropriation dans l'acquisition de la langue maternelle. AILE, 2, 143-171.

Musiol, M., Trognon, A., Coulon, D., \& Bocéréan, C. (2006). Structure de l'Interaction Verbale et Rationalité Argumentative chez l'Enfant Multiplyhandicapé : Répétitions, Reprises et Dynamique. Le Langage et l'Homme, XXXXI, 2, 27-43.

Nelson, K. E., Welsh, J. M., Camarata, S. M., Tjus, T. \& Heimann, M. (2001). A rare event transactional model of tricky mix conditions contributing to language acquisition and varied communicative delays. In K. E. Nelson, A. Aksu-Koç, \& C. E. Johnson (eds), Children's language, Vol. 11 (165-195). Hillsdale-NJ: Lawrence Erlbaum Associates.

Perrin, L., Deshaies, D., \& Paradis, C. (2003). Pragmatic functions of local diaphonic répétition in conversation. Journal of Pragmatics, 35, 1843-1860.

Rieger, C. L. (2003). Répétition as auto-repair strategies in English and German conversations. Journal of Pragmatics, 35, 47-69.

Roulet, E., Auchlin, A., Moeschler, J., Rubattel, C., \& Schelling, M. (1985). L'articulation du discours en français contemporain. Berne : Peter Lang.

Salazar-Orvig, A. (2000). La reprise aux sources de la construction discursive. Langage, 140, 68-91.

Schegloff, E. A., Jefferson, G., \& Sachs, H. (1977). The preference for auto-correction in the organization of repair in conversation. Language, 53, 361-382.

Searle, J. R. (1969/1972). Les actes de langage. Hermann : Paris.

Searle, J. R. \& Vanderveken, D. (1985). Foundations of illocutionary logic. Cambridge : C.P.U.

Snow, C. E. (1995). Issues in the Study of Input : Finetuning, Universality, Individual and Developmental Differences, and Necessary Causes. In P. Fletcher, \& B. MacWhinney (eds.), The handbook of child language (180-193). Oxford: Blackwell Publishing.

Trognon, A., \& Brassac, C. (1992). L'enchaînement conversationnel. Cahiers de linguistique française, 13, 67-108.

Vanderveken, D., \& Susumo, K. (2002). Introduction. In D. Vanderveken, \& S. Kubo (eds), Essays in speech acts theory (1-21). Amsterdam: John Benjamins Publishing Company. 
Veneziano, E. (2000). Interaction, conversation et acquisition du langage dans les trois premières années. In M. Kail, \& M. Fayol (eds), L'acquisition du langage. Le langage en émergence de la naissance à trois ans (231-265). Paris : P.U.F.

Verhaegen, F., Bocéréan, C. \& Musiol, M. (2008). Rationalité du processus de répétition chez l'enfant normal et multiplyhandicapé : aux frontières de la pathologie. Philosophia Scientiae, 12 (2), 111128.

Vigil, D., C., Hodges, J., \& Kle, T. (2005). Quantity and quality of parental language input to latetalking todlers during play. Child Language Teaching \& Therapy, 21 (2), 107-122.

Yont, K., M., Hewitt, L., R., \& Miccio, A., W. (2002). What dit you say?: Understanding conversational breakdown in children with speech and language impairments. Clinical Linguistics and Phonetics, 16 (4), 265-28. 